\title{
Expanding the Scope of Electronic-Structure Theory
}

\author{
An efficient new approach makes density-functional simulations feasible \\ over larger length scales.
}

\author{
By Rex Godby
}

$\square$ ensity-functional theory (DFT) has, since the 1970s, had a huge impact on our understanding of condensed-matter physics through its ability to describe the effect of the electrons' mutual interaction on the electronic structure of matter. However, in solids in which successive crystal unit cells are no longer exact repetitions of one another, the usual approach for implementing DFT can run out of steam. Now, Tristan Müller at the Max Planck Institute of Microstructure Physics, Germany, and colleagues have devised an efficient new way to implement DFT in the presence of such a long-wavelength variation [1]. Their technique potentially extends the scope of DFT to encompass phenomena of technological interest, such as skyrmions and magnetic domain walls.

The key that unlocks a material's electronic structure is an almost magical result known as Bloch's theorem, which greatly assists the solution of Schrödinger's equation [2]. Rather than having to take account of arbitrary mixing of the atomic wave functions of the solid's infinite number of atoms, Bloch showed that the atoms in each unit cell of the solid contribute equally to any wave function. The contribution of each unit cell differs from its neighbor's only by a phase factor that is a fixed characteristic of a given wave function. This phase factor is normally described through a Bloch wave vector, the "k point." In essence, this idea means that solving Schrödinger's equation for electrons in a periodic solid is little costlier than solving it for a single unit cell. The efficiency of this approach facilitated the development of electronic-structure calculations for solids in the early decades of quantum mechanics [3].

The first such calculations did not take into account the effect of the electrostatic repulsion between electrons on the material's electronic structure. Correcting this shortcoming is where DFT comes in $[4,5]$. In DFT, a Schrödinger equation is still solved for each electron in turn, but with the periodic potential felt by the electrons now modified by the periodic density of the electrons themselves within each unit cell. Crucially, the power of Bloch's theorem is preserved. The combination of quantitative accuracy and efficiency fueled the explosion in applications of DFT to crystalline solids from the 1970 s onwards [6].

What if the system under study is not a periodic solid but is nevertheless infinite? Often, the concept of a "supercell" is useful-a larger unit cell within which a periodic atomic arrangement can still be assumed to a good approximation. (The simplest example would be an antiferromagnetic material, in which the alternating spins of neighboring atoms double the periodicity.) The power of Bloch's theorem is then regained, albeit with increased computational cost reflecting the presence of, perhaps, dozens of atoms in the new unit cell rather than just one or two. The cost typically scales with the cube of this number of atoms, so supercell calculations can be very (even prohibitively) costly. If, for example, the supercell is 10 times larger than the basic unit cell in each direction, then the reciprocal lattice becomes 10 times finer in each direction. This expansion greatly increases the number of coefficients that must be calculated for each electronic wave function and for the corresponding DFT potentials.

Tackling this scaling problem is the purpose of the new work by Müller and colleagues. To achieve their goal, the researchers developed a flexible approach that is closely related to the concept of "satellite" peaks in x-ray crystallography and electron diffraction. There, the observed image is a series of diffraction peaks that is essentially the Fourier transform of the 


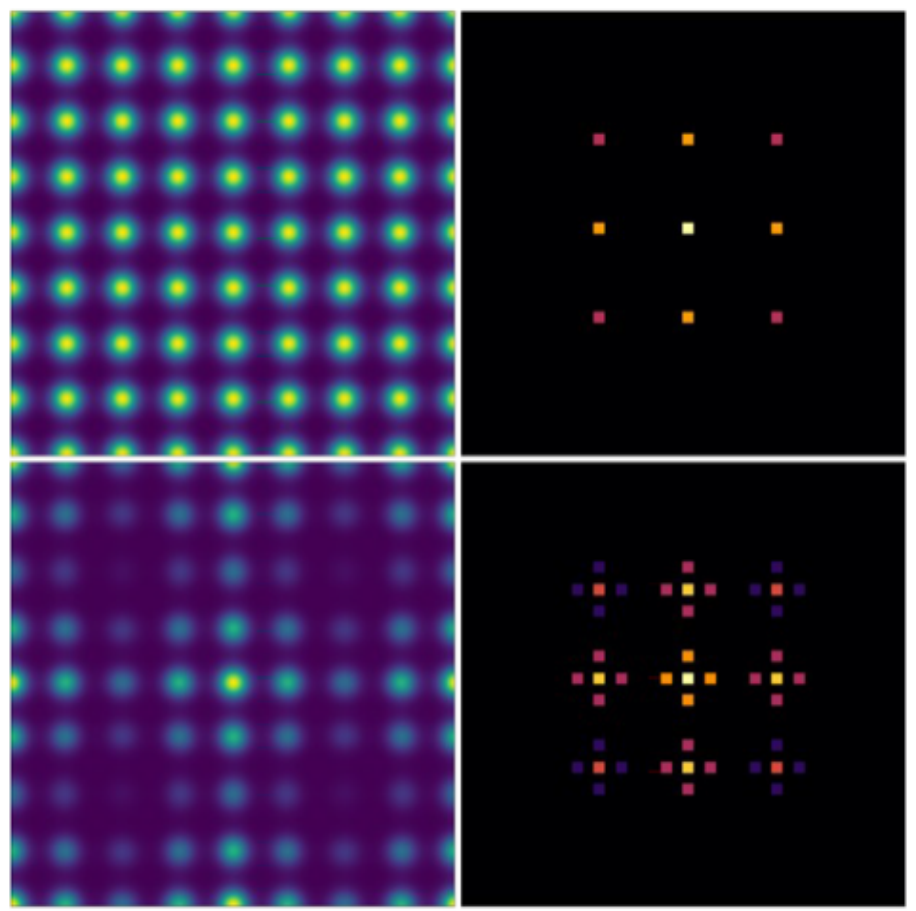

Figure 1: The diffraction patterns on the right are the Fourier transforms of the crystal structures on the left. (Top) A normal periodic solid. (Bottom) A spatially modulated version of the solid: each diffraction peak on the right becomes decorated with a small number of "satellite" peaks that reflect the nature of the spatial modulation. The new method effectively harnesses this concept of satellite peaks to give an efficient way of calculating and storing the electron wave functions, charge density, and magnetization density of a modulated solid, especially when the modulation is on a long length scale.

Credit: R. Godby/University of York

structure of the solid under observation. If the solid is "modulated" by acquiring a new, longer periodicity, then each diffraction peak becomes surrounded by a finely spaced set of a few satellite peaks (Fig. 1).

Away from each original peak position, the intensity of the satellites falls off quickly, provided that the modulation has a long wavelength. In the language of a supercell DFT calculation, this behavior means that much information can be neglected to a good approximation: only a few satellite coefficients need be calculated in place of each original coefficient (that describe the electronic wave function or charge or magnetization density for the original periodic solid).

Müller and colleagues, then, address the situation of a periodic solid upon which an additional spatial variation on a long length scale is imposed-either an externally applied potential or a spontaneous internal adjustment of the electrons themselves, such as a charge-density wave. Their approach is equivalent to a DFT supercell calculation plus certain well-founded approximations arising from the retention of a limited set of satellite coefficients, which is the key to the efficiency. As is common in DFT, the local electronic structure is represented using a compact set of functions within each unit cell. Meanwhile, the satellite aspects of the wave functions and densities are naturally described using long-wavelength plane waves, which allows these parts of the calculation to benefit from the numerical efficiency of fast Fourier transforms.

As a demonstration of their technique, Müller and colleagues present three examples: the spin-spiral state of the $\gamma$ phase of Fe; coupled spin and charge-density waves in $\mathrm{Cr}$; and LiF with an externally applied potential. It is noteworthy that their method need not start from any rigid assumption about the modulation of the original solid, other than that it is on a length scale of many unit cells. When the electronic ground state, as given by DFT, is found, the nature of the modulation (spin spiral or charge-density wave, for example) emerges naturally from the calculation.

When the researchers compare their model's results with full supercell calculations, it is clear that the two methods are not yet in perfect alignment. However, given sufficient computer power, this mismatch should narrow. Looking beyond materials' electronic ground states, Müller and colleagues foresee the application of their approach to the time dependence of such modulated solids, making use of time-dependent DFT [7]. This ability should enable the $a b$ initio simulation of the dynamic coupling between the electronic wave functions on an atomic scale with, say, electromagnetic waves on a longer length scale in a plasmonic optoelectronic device. For designers of such nanostructures, the electromagnetic waveforms emitted in response to some intense applied pulse could therefore take proper account of the quantum-mechanical motion of the electrons, without the limitations of perturbation theory. 
Rex Godby: Department of Physics, University of York, York, United Kingdom

\section{REFERENCES}

1. T. Müller et al., "Extending solid-state calculations to ultra-long-range length scales," Phys. Rev. Lett. 125, 256402 (2020).

2. F. Bloch, "On the quantum mechanics of electrons in crystal lattices," Z. Phys. 52, 555 (1929).

3. N. F. Mott and H. Jones, The Theory of the Properties of Metals and Alloys (Clarendon Press, Oxford, 1936).

4. P. Hohenberg and W. Kohn, "Inhomogeneous electron gas," Phys. Rev. 136, B864 (1964).

5. W. Kohn and L. J. Sham, "Self-consistent equations including exchange and correlation effects," Phys. Rev. 140, A1133 (1965).

6. O. Krogh Andersen, "Linear methods in band theory," Phys. Rev. B 12, 3060 (1975).

7. E. Runge and E. K. U. Gross, "Density-functional theory for time-dependent systems," Phys. Rev. Lett. 52, 997 (1984). 